\title{
Colonization with multidrug-resistant Enterobacteriaceae among infants: an observational study in southern Sri Lanka
}

\author{
Hannah R. Meredith ${ }^{1,2^{*}}$ (D), Sarath Kularatna ${ }^{3}$, Kristin Nagaro ${ }^{4}$, Ajith Nagahawatte ${ }^{5,6}$, Champica Bodinayake ${ }^{6,7}$, \\ Ruvini Kurukulasooriya ${ }^{5}$, Nishadhi Wijesingha ${ }^{5}$, Lyndy B. Harden ${ }^{13}$, Bhagya Piyasiri ${ }^{8}$, Amr Hammouda ${ }^{11}$, Brian \\ M. Wiegmann ${ }^{9}$, Bradly P. Nicholson ${ }^{10}$, Maria Joyce ${ }^{4,11}$, Christopher W. Woods ${ }^{4,6,11}$, Arnoud H. M. Van Vliet ${ }^{12}$, \\ Siddhartha Thakur ${ }^{13,14}$ and L. Gayani Tillekeratne ${ }^{4,6,7,11^{*}}$
}

\begin{abstract}
Background: The timing of and risk factors for intestinal colonization with multidrug-resistant Enterobacteriaceae (MDRE) are still poorly understood in areas with high MDRE carriage. We determined the prevalence, timing, and risk factors associated with MDRE intestinal colonization among infants in southern Sri Lanka.

Methods: Women and their newborn children were enrolled within $48 \mathrm{~h}$ after delivery in southern Sri Lanka. Rectal swabs were collected from women and infants at enrollment and 4-6 weeks later. Enterobacteriaceae were isolated and identified as MDRE (positive for extended-spectrum $\beta$-lactamases or carbapenem resistant) using standard microbiologic procedures. We used exact methods (Fisher's exact and Kruskal-Wallis tests) and multivariable logistic regression to identify sociodemographic and clinical features associated with MDRE intestinal colonization. Whole-genome sequencing was performed on selected MDRE isolates to identify phylogroups and antibiotic resistance-encoding genes were identified with NCBI's AMRfinder tool.
\end{abstract}

Results: Overall, 199 post-partum women and 199 infants were enrolled; 148/199 (74.4\%) women and 151/199 (75.9\%) infants were reassessed later in the community. Twenty-four/199 (12.1\%) women and 3/199 (1.5\%) infants displayed intestinal colonization with MDRE at enrollment, while 26/148 (17.6\%) women and 24/151 (15.9\%) infants displayed intestinal colonization with MDRE at the reassessment. While there were no risk factors associated with infant colonization at enrollment, multivariable analysis indicated that risk factors for infant colonization at reassessment included mother colonized at enrollment $(\mathrm{aOR}=3.62)$ or reassessment $(\mathrm{aOR}=4.44)$, delivery by Cesarean section $(a O R=2.91)$, and low birth weight $(a O R=5.39)$. Of the 20 MDRE isolates from infants that were sequenced, multilocus sequence typing revealed that 6/20 (30\%) were clustered on the same branch as MDRE isolates found in the respective mothers. All sequenced isolates for mothers (47) and infants (20) had at least one ESBL-producing gene. Genes encoding fosfomycin resistance were found in 33/47 (70\%) of mothers' isolates and 16/20 (80\%) of infants' isolates and genes encoding resistance to colistin were found in one (2\%) mother's isolate.

\footnotetext{
*Correspondence: hmeredi4@jhu.edu; gayani.tillekeratne@duke.edu

1 Department of Biomedical Engineering, Duke University, Durham, NC,

USA

${ }^{4}$ Division of Infectious Diseases, Department of Medicine, Duke

University School of Medicine, Durham, NC, USA

Full list of author information is available at the end of the article
}

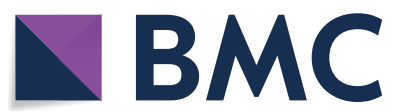

(c) The Author(s) 2021. Open Access This article is licensed under a Creative Commons Attribution 4.0 International License, which permits use, sharing, adaptation, distribution and reproduction in any medium or format, as long as you give appropriate credit to the original author(s) and the source, provide a link to the Creative Commons licence, and indicate if changes were made. The images or other third party material in this article are included in the article's Creative Commons licence, unless indicated otherwise in a credit line to the material. If material is not included in the article's Creative Commons licence and your intended use is not permitted by statutory regulation or exceeds the permitted use, you will need to obtain permission directly from the copyright holder. To view a copy of this licence, visit http://creativecommons.org/licenses/by/4.0/. The Creative Commons Public Domain Dedication waiver (http://creativeco mmons.org/publicdomain/zero/1.0/) applies to the data made available in this article, unless otherwise stated in a credit line to the data. 
Conclusions: Our results suggest that a substantial proportion of infants undergo MDRE intestinal colonization within 6 weeks of birth, potentially due to postnatal rather than intranatal transmission.

Keywords: ESBL, CRE, Multidrug-resistant Enterobacteriaceae, Sri Lanka, Intestinal colonization

\section{Introduction}

Infections due to multidrug-resistant Enterobacteriaceae (MDRE), such as extended-spectrum $\beta$-lactamase-producing and carbapenem-resistant Enterobacteriaceae (ESBL-E and CRE, respectively), are increasing in prevalence worldwide [1]. These infections are difficult to treat and associated with high morbidity and mortality [2]. The rise in ESBL-E and CRE infections has been more pronounced in regions such as the Middle East and South Asia, where a large proportion of otherwise healthy people (up to 70\%) can asymptomatically carry ESBL-E or CRE in their gastrointestinal tracts [3, 4]. Since colonization is a precursor to infections such as bacteremia, pneumonia, and urinary tract infection, preventing MDRE colonization is an infection control priority.

In communities that have high baseline MDRE intestinal colonization prevalence, intranatal transmission (i.e., transmission at birth) or postnatal transmission (i.e., transmission through breastmilk, environment-toperson exposure, or person-to-person exposure after birth) may play an important role in MDRE intestinal colonization of infants. Limited available data have identified both intranatal risk factors, such as maternal colonization, and postnatal risk factors, such as water sources and pet exposure, for MDRE colonization [5-7]. Few of these published studies have been conducted in South Asia, where MDRE intestinal colonization prevalence is high (30-70\% in South Asian countries as compared with $<15 \%$ in Europe) [3, 8]. In addition, conflicting conclusions exist as to whether exposures such as antibiotic use are risk factors for MDRE colonization [5, 9, 10].

In Sri Lanka, a south Asian country, the prevalence of MDRE intestinal colonization, age at which colonization occurs, and risk factors for colonization are unknown. We conducted an exploratory, prospective study in southern Sri Lanka to identify the prevalence, timing, and risk factors associated with MDRE intestinal colonization among infants in southern Sri Lanka.

\section{Methods}

\section{Study design}

The study was conducted in the post-partum wards of Mahamodara Teaching Hospital, the largest (400-bed) obstetric/gynecologic hospital in Southern Province, Sri Lanka, from August 2016 to January 2017.

\section{Study cohort and enrollment}

Women ( $\geq 18$ years old) who had presented in labor were approached within $48 \mathrm{~h}$ of delivery regarding study participation. Women who lived $>40 \mathrm{~km}$ from the hospital or who had active rectal bleeding were excluded. One rectal sample was collected from each woman and from each neonate using a FecalSwab (Copan Diagnostics) with Cary Blair media. If a patient was unable to tolerate rectal sampling, either a peri-rectal or fresh stool sample was collected using the swab. A standardized questionnaire was administered to collect sociodemographic information, clinical data, and exposure history. The medical record was reviewed during hospitalization to obtain information about the clinical course, such as receipt of antibiotics and clinical outcomes.

\section{Reassessment}

Patients were visited at home 4-6 weeks after discharge to collect one rectal sample from each woman and infant using a method identical to that used at enrollment. A standardized questionnaire was administered to collect further details about exposures within the home environment.

\section{Microbiologic testing}

Each swab was streaked on MacConkey agar with $2 \mu \mathrm{g} /$ $\mathrm{mL}$ cefotaxime. Plates were incubated at $37{ }^{\circ} \mathrm{C}$ for $48 \mathrm{~h}$ and up to 3 morphologically different isolates per sample were selected for further characterization. Isolates were stored at $-70{ }^{\circ} \mathrm{C}$ until further analysis.

Species-level identification was performed using matrix-assisted laser desorption/ionization-time of flight mass spectrometry (MALDI-TOF, Bruker, Billerica, MA, USA). For organisms identified as Enterobacteriaceae, antibiotic susceptibilities and minimum inhibitory concentrations (MICs) were determined using MicroScan technology (Beckman Coulter, CA, USA), which automates organism identification and antimicrobial susceptibility testing results using microbroth dilution, according to Clinical and Laboratory Standards Institute guidelines [11]. Minimum inhibitory concentrations, as determined by microbroth dilution, were used to verify ESBL production. A decrease of $\geq 3$ doubling dilutions in the MIC for either cefotaxime or ceftazidime tested in combination with $4 \mu \mathrm{g} / \mathrm{mL}$ clavulanic acid, versus its MIC when tested alone, was considered indicative of ESBL production. 
A modified Hodge test was performed on isolates with an intermediate or resistant susceptibility profile to ertapenem to detect the presence of carbapenemases. Phenotypic resistance to colistin and fosfomycin were confirmed by microbroth dilution, where a MIC $>4 \mu \mathrm{g} /$ $\mathrm{mL}$ was considered indicative of colistin resistance and MIC $>128 \mu \mathrm{g} / \mathrm{mL}$ was considered indicative of fosfomycin resistance.

\section{Genome library preparation and sequence assembly}

Whole genome sequencing of Escherichia coli isolates was conducted as previously described [12]. Briefly, the E. coli isolates $(\mathrm{n}=76)$ were cultured overnight at $37^{\circ} \mathrm{C}$ in Luria-Bertani media. Genomic DNA was extracted using a MasterPure Gram Positive DNA Purification Kit (Lucigen). DNA concentrations were quantitated using a Qubit 3.0 Fluorometer for double-stranded-DNA highsensitivity assay kit (Thermo Fisher Scientific). The DNA was normalized and Illumina Nextera XT was used to prepare libraries: indexed libraries were generated using 12 cycles of PCR and each sample was indexed with a unique barcode. PCR cleanup and fragment size exclusion were done with an Ampure XP bead cleanup protocol to capture the desired band sizes for the DNA library (from $250 \mathrm{bp}$ to $1.0 \mathrm{~kb}$ ). The fragmented DNA library was manually normalized and 24 libraries were pooled equally based on molar concentration and sequenced on the Illumina MiSeq v2 500 (2*250 bp paired-end reads) platform (MiSeq reagent kit, version 3). A PhiX control was spiked into each run as a quality control (QC). QC metrics monitored included clusters passing filter, base call quality scores (Q-Scores), cluster density, and estimated yields for each library and the PhiX control. FastQ files were generated and transferred to the NCBI SRA repository.

\section{Genome assembly and genomic analyses}

FastQ files were obtained from the NCBI SRA repository using fastq-dump included in the SRA toolkit (https:// www.ncbi.nlm.nih.gov/sra/docs/toolkitsoft/) (see SRA accession numbers in Additional File 1: Table S5). Genome sequences were assembled using Shovill version 1.0.4 (https://github.com/tseemann/shovill) using the SPAdes assembler with a coverage cut-off of $2 \times$ and minimum contig size of $200 \mathrm{nt}$ [13]. Assembly metrics were obtained using Quast version 4.6 [14]. The genome sequences were further characterized by determining the multilocus sequence type (ST) using MLST version 2.17.6 (https://github.com/tseemann/mlst) and the Warwick 7-gene scheme [15]. Serotypes were predicted using Abricate version 0.9.8 (https://github.com/tseem ann/abricate) and the SerotypeFinder database [16]. The phylogroup of each isolate was determined using the
ClermonTyping program version 1.4.1 [17]. Prediction of antimicrobial resistance was done using the Abricate program with the NCBI Bacterial Antimicrobial Resistance Reference Gene Database and the Resfinder database, and confirmed using AMRfinder version v3.1.1b $[18,19]$. Phylogenetic trees were based on core genome single-nucleotide polymorphisms as identified using ParSNP version 1.2 using settings described previously $[12,20]$. The final genomic sequences were uploaded to the designated NCBI Bioproject (PRJNA293225).

\section{Statistical analysis}

Clinical data were entered into a Microsoft Access (Version 1808) database. To identify demographic features associated with MDRE intestinal colonization, Fisher's exact and Kruskal-Wallis analyses were conducted for categorical and continuous data, respectively. Multivariable logistic regression analysis was also conducted to identify risk factors associated with MDRE intestinal colonization in infants and in mothers. Any risk factors associated with intestinal colonization with a $p$ value $<0.05$ on bivariable analysis were included in the multivariable model. Variables were checked for collinearity prior to inclusion in the model. As antibiotic use in mothers and delivery mode were collinear, antibiotic use in mothers was dropped from the multi-variable model for mothers. To create a more parsimonious model, risk factors were excluded in a step-wise manner until all $p$ values were $<0.05$. Each excluded variable was then added sequentially to ensure that it was not significant in the final model. Statistical analyses were conducted in STATA (Version 15.0).

\section{Ethical considerations}

Ethical approval for this study was obtained from the Faculty of Medicine, University of Ruhuna, Ethical Review Committee (Sri Lanka) and the Duke University Institutional Review Board (USA).

\section{Results \\ Study cohort}

A total of 200 women and 202 infants (two sets of twins) were enrolled; however, samples from one woman and three infants were compromised in the storage process, thus leaving 199 women and 199 infants from enrollment for analysis. The median age for women was 29.0 years (IQR 25-33 years) and 95/199 (47.7\%) of infants were female (Table 1, Additional File 1: Table S1). Most (85.4\%) women were housewives or unemployed, and the majority $(87.4 \%)$ had completed at least grade 10 education. The majority of women (158/199) reported a monthly household income ranging from 15,000 to 45,000 Rs (approximately 100-310 USD; Sri Lanka's household 
Table 1 Sociodemographic and clinical features of infants at enrollment and reassessment

\begin{tabular}{|c|c|c|c|c|c|c|c|c|}
\hline & \multicolumn{4}{|c|}{ Infant at admission } & \multicolumn{4}{|c|}{ Infant at follow-up } \\
\hline & All $(n=199)$ & MDRE $+(n=3)$ & MDRE- $(n=196)$ & $p$ value & All $(n=151)$ & $\mathrm{MDRE}+(\mathrm{n}=24)$ & MDRE- $(n=127)$ & $p$ value \\
\hline \multicolumn{9}{|l|}{ General details } \\
\hline Mother's age (year) & $29(25-33)$ & $32(20-34)$ & $29(25-33)$ & 0.749 & $29(26-33)$ & $30(25-34)$ & $29(26-33)$ & 0.129 \\
\hline $\begin{array}{l}\text { Infant's gender } \\
\text { (female) }\end{array}$ & $95(47.7)$ & $2(66.7)$ & $93(47.5)$ & 0.614 & $71(47.0)$ & $10(41.7)$ & $61(48.0)$ & 0.365 \\
\hline $\begin{array}{l}\text { Low birth weight } \\
(<2.5 \mathrm{~kg})\end{array}$ & $19(9.6)$ & $0(0)$ & $19(9.69)$ & 1.000 & $16(10.6)$ & $6(25)$ & $10(7.9)$ & 0.023 \\
\hline Breastfed & $198(99.5)$ & $3(100)$ & 195 (99.5) & 0.985 & $151(100)$ & $24(100)$ & $127(100)$ & 1.000 \\
\hline $\begin{array}{l}\text { Average monthly } \\
\text { household income }\end{array}$ & 0.124 & & & & 0.657 & & & \\
\hline$<15,000$ Rs & $10(5.0)$ & $0(0)$ & $10(5.1)$ & & $9(6.0)$ & $0(0)$ & $9(7.1)$ & \\
\hline $15,001-30,000$ Rs & 77 (38.7) & $1(33.3)$ & $76(38.8)$ & & $58(38.4)$ & $11(45.8)$ & $47(37.0)$ & \\
\hline $30,001-45,000$ Rs & $82(41.2)$ & $0(0)$ & $82(41.8)$ & & $62(41.1)$ & $10(41.7)$ & $52(41.0)$ & \\
\hline$>45,000 \mathrm{Rs}$ & $30(15.1)$ & $2(66.7)$ & $28(14.3)$ & & $22(14.6)$ & $3(12.5)$ & $19(15.0)$ & \\
\hline \multicolumn{9}{|c|}{ Mother's hospital-associated risk factors (within 6 months prior to delivery) } \\
\hline Hospitalization & $37(18.6)$ & $0(0)$ & $37(18.9)$ & 0.538 & $26(17.2)$ & $2(8.33)$ & $24(18.9)$ & 0.169 \\
\hline History of infection & $9(4.5)$ & $0(0)$ & $9(4.6)$ & 0.870 & $9(6.0)$ & $1(4.2)$ & $8(6.3)$ & 0.566 \\
\hline Antibiotic intake & $6(3.0)$ & $1(33.3)$ & $5(2.6)$ & 0.088 & $6(4.0)$ & $1(4.2)$ & $5(3.9)$ & 0.653 \\
\hline \multicolumn{9}{|c|}{ Household-associated risk factors (within 6 months prior to delivery) } \\
\hline $\begin{array}{l}\text { Number of adults at } \\
\text { home }\end{array}$ & $2(1-3)$ & $3(1-4)$ & $2(1-3)$ & 0.95 & $2(1-3)$ & $2(1.5-3)$ & $2(1-3)$ & 0.266 \\
\hline $\begin{array}{l}\text { Number of children at } \\
\text { home }\end{array}$ & $1(1-2)$ & $1(1-1)$ & $1(1-2)$ & 0.64 & $1(1-2)$ & $1(0-2)$ & $1(1-2)$ & 0.214 \\
\hline \multicolumn{9}{|c|}{ Complications during pregnancy } \\
\hline Any complications & $22(11.1)$ & $0(0)$ & $22(11.2)$ & 0.702 & $20(13.3)$ & $5(20.8)$ & $15(11.8)$ & 0.189 \\
\hline $\begin{array}{l}\text { Premature rupture of } \\
\text { membranes }\end{array}$ & $5(2.51)$ & $0(0)$ & $5(2.6)$ & 0.926 & $5(3.3)$ & $2(8.3)$ & $3(2.4)$ & 0.179 \\
\hline Gestational diabetes & $6(3.02)$ & $0(0)$ & $6(3.1)$ & 0.912 & $6(4.0)$ & $2(8.3)$ & $4(3.2)$ & 0.243 \\
\hline Labor induced & $13(6.5)$ & $0(0)$ & $13(6.6)$ & 1.000 & $11(7.3)$ & $1(4.2)$ & $10(7.9)$ & 1.000 \\
\hline Mode of delivery & & & & 0.559 & & & & 0.021 \\
\hline C-section & $64(32.2)$ & $0(0)$ & $64(32.7)$ & & $51(33.6)$ & $13(54.2)$ & $38(29.9)$ & \\
\hline Vaginal & $134(67.3)$ & $3(100)$ & $131(66.8)$ & & $100(66.2)$ & $11(45.8)$ & $89(70.1)$ & \\
\hline $\begin{array}{l}\text { Difficulties with } \\
\text { delivery }\end{array}$ & & & & 0.592 & & & & 0.133 \\
\hline No & $62(31.2)$ & $0(0)$ & $62(31.6)$ & & $49(32.5)$ & $13(54.2)$ & $36(28.4)$ & \\
\hline Episiotomy & $131(65.8)$ & $3(100)$ & $128(65.3)$ & & $97(64.2)$ & $11(45.8)$ & $86(67.7)$ & \\
\hline \multicolumn{9}{|c|}{ Hospitalization/discharge details } \\
\hline NICU admission & & & & & $3(2.0)$ & $1(4.2)$ & $2(1.6)$ & 0.407 \\
\hline $\begin{array}{l}\text { Antibiotic started in } \\
\text { hospital }\end{array}$ & & & & & $3(2.0)$ & $1(4.2)$ & $2(1.6)$ & 0.407 \\
\hline $\begin{array}{l}\text { Hospital stay—Infant } \\
\text { (days) }\end{array}$ & & & & & $1(1-2)$ & $2(1-3)$ & $1(1-2)$ & 0.060 \\
\hline \multicolumn{9}{|l|}{ Infant reassessment } \\
\hline $\begin{array}{l}\text { Admitted to hospital } \\
\text { again }\end{array}$ & & & & & $7(4.6)$ & $1(4.2)$ & $6(4.7)$ & 0.692 \\
\hline $\begin{array}{l}\text { In-patient antibiotic } \\
\text { use }\end{array}$ & & & & & $4(2.7)$ & $0(0)$ & $4(3.2)$ & 1.000 \\
\hline $\begin{array}{l}\text { Out-patient antibiotic } \\
\text { use }\end{array}$ & & & & & $21(13.9)$ & $6(25)$ & $15(11.81)$ & 0.087 \\
\hline \multicolumn{9}{|l|}{ Housing details } \\
\hline Refrigerator & & & & & $102(67.6)$ & $15(62.5)$ & $87(68.5)$ & 0.361 \\
\hline Running water & & & & & $140(92.7)$ & $22(91.7)$ & $118(92.9)$ & 0.549 \\
\hline
\end{tabular}


Table 1 (continued)

\begin{tabular}{|c|c|c|c|c|c|c|c|c|}
\hline & \multicolumn{4}{|c|}{ Infant at admission } & \multicolumn{4}{|c|}{ Infant at follow-up } \\
\hline & All $(n=199)$ & MDRE $+(n=3)$ & MDRE- $(n=196)$ & $p$ value & All $(n=151)$ & MDRE + $(n=24)$ & MDRE- $(n=127)$ & $p$ value \\
\hline Toilet type & & & & & & & & 0.199 \\
\hline $\begin{array}{l}\text { Outdoor squat toi- } \\
\text { let-private }\end{array}$ & & & & & $116(76.8)$ & $23(95.8)$ & $93(73.2)$ & \\
\hline $\begin{array}{l}\text { Outdoor squat toi- } \\
\text { let—public }\end{array}$ & & & & & $32(21.2)$ & $1(4.2)$ & $31(24.4)$ & \\
\hline $\begin{array}{l}\text { Private toilet/com- } \\
\text { mode }\end{array}$ & & & & & $2(1.3)$ & $0(0)$ & $2(1.6)$ & \\
\hline Pets at home & & & & & $83(55.0)$ & $15(62.5)$ & $68(53.5)$ & 0.653 \\
\hline $\begin{array}{l}\text { Household water treat- } \\
\text { ment methods }\end{array}$ & & & & & & & 0.204 & \\
\hline Boiled & & & & & $67(44.4)$ & $9(37.5)$ & $58(45.7)$ & \\
\hline Filtered & & & & & $21(13.9)$ & $5(20.8)$ & $16(12.6)$ & \\
\hline None & & & & & $68(45.0)$ & $9(37.5)$ & $59(46.5)$ & \\
\hline \multicolumn{9}{|l|}{ Colonization } \\
\hline Mother at enrollment & $24(12.1)$ & $2(66.7)$ & $22(11.2)$ & 0.054 & $19(12.6)$ & $7(29.2)$ & $12(9.5)$ & 0.026 \\
\hline $\begin{array}{l}\text { Mother at reassess- } \\
\text { ment }\end{array}$ & & & & & $26(17.2)$ & $9(37.5)$ & $17(13.4)$ & 0.012 \\
\hline Infant at enrollment & & & & & $3(2.0)$ & $2(8.3)$ & $1(0.8)$ & 0.051 \\
\hline
\end{tabular}

Risk factors associated with intestinal colonization with multidrug resistant Enterobacteriaceae (MDRE) on bivariable analysis are shown Values are reported as frequency (\%) or median (interquartile range). See Additional File 1: Table S1 for equivalent information regarding mothers a $<15,000$ Rs $\leq 89$ USD; 15,001-30,000 Rs = 89-177 USD; 30,001-45,000 Rs =177-266; $>45,000$ Rs $\geq 266$ USD

average monthly income per capita was 115 USD in 2016) [21]. Reassessments were completed for 149/199 (75.4\%) women and 151/199 (75.9\%) infants; however, the sample from one woman was compromised during the storage process, thus leaving 148 women from reassessment for analysis. The median time between enrollment and the reassessment was 5.9 weeks (IQR 4.9-7.0 weeks) after hospital discharge.

There were complications in 21/199 (10.6\%) pregnancies: $4 / 199$ (2.0\%) had pre-mature rupture of membranes, $6 / 199$ (3.0\%) had gestational diabetes, and 13/199 (6.5\%) had labor induced. Sixty-two/199 (31.2\%) women underwent Caesarean section (C-section) and received prophylactic antibiotics prior to the procedure. In addition, $1 / 199(0.5 \%)$ mother received antibiotics for treatment of infection during hospitalization. The median gestation period was 38.0 weeks (IQR 38.0-39.0 weeks). Nineteen/199 (9.6\%) infants had low birth weight $(<2.5 \mathrm{~kg})$. Most infants were breastfed at the time of enrollment (198/199, 99.5\%). Antibiotics (including amoxicillin, a third-generation cephalosporin, penicillin, an aminoglycoside, and penicillin) were administered to 3/199 (1.5\%) infants during hospitalization.

\section{MDRE intestinal colonization prevalence}

Of the total cohort, rectal swabs from $40 / 199$ (20.1\%) mothers and 16/199 (8.0\%) infants at enrollment and
41/148 (27.7\%) mothers and 46/151 (30.5\%) infants at reassessment resulted in growth of colonies on MacConkey with cefotaxime media. Of the isolates that grew on selective media, $3 / 40(7.5 \%)$ of samples derived from mothers and $3 / 16(18.8 \%)$ of samples derived from infants at enrollment and 4/41 (9.8\%) of mothers' and 12/46 (26.1\%) of infants' samples at the reassessment could not be re-cultured from storage, likely due to decreased viability associated with a freeze-thaw cycle, and were considered not to be MDRE for the purposes of the primary analysis. A sensitivity analysis in which these samples were evaluated as MDRE was conducted and showed no significant changes to the risk factors later discussed (Additional File 1: Table S2). Upon further analysis of the remaining isolates that grew on selective media, isolates from 12/40 (30\%) of mothers at enrollment, 7/16 (43.8\%) infants at enrollment, 10/41 (24.4\%) of mothers at reassessment, and 10/46 (21.7\%) of infants at reassessment were identified by MicroScan and MALDI-TOF as nonESBL and non-CRE producing bacteria and thus categorized as non-MDRE. Only patients for whom isolates could be fully characterized as being MDRE were identified as being colonized with MDRE in the subsequent analyses.

Overall, 24/199 (12.1\%) women and 3/199 (1.5\%) infants had MDRE detected at enrollment, while 26/148 (17.6\%) women and 24/151 (15.9\%) infants had MDRE 


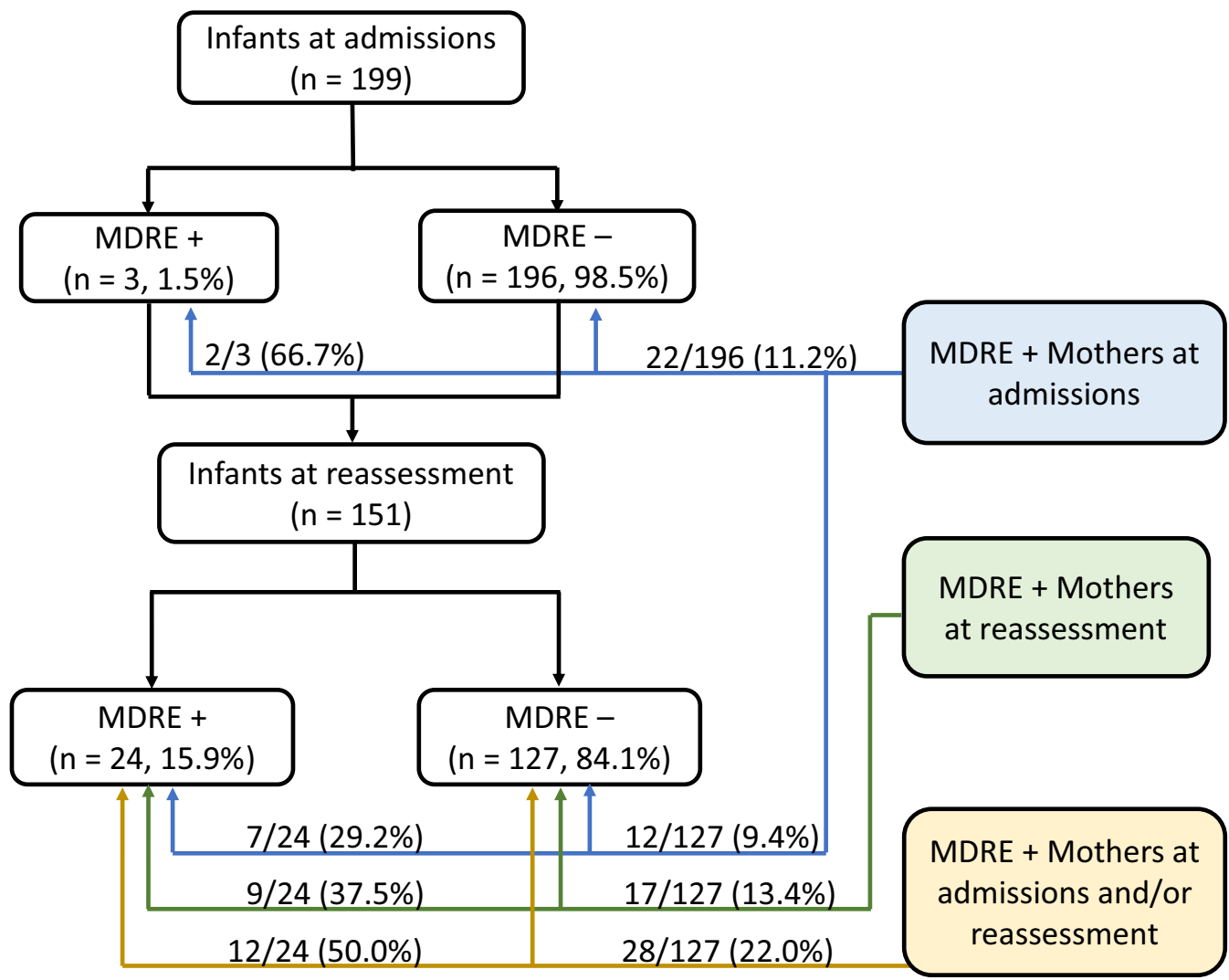

Fig. 1 Flow chart of mothers and infants colonized with multidrug resistant Enterobacteriaceae (MDRE) at enrollment and reassessment. Counts inside the boxes represent the total number of infants for that category. Counts (proportion) on the lines represent the infants in that category who had MDRE + mothers at admissions (blue), reassessment (green), or admissions and/or reassessment (yellow)

Table 2 Prevalence of multidrug-resistant Enterobacteriaceae (MDRE) intestinal colonization in mothers and infants

\begin{tabular}{lcc}
\hline & Mother (\%) & Infant (\%) \\
\hline Enrollment $\left(\mathrm{n}_{\text {mother }}=199, \mathrm{n}_{\text {infant }}=199\right)$ & $24(12.1)$ & $3(1.5)$ \\
ESBL E. coli & $22(91.7)$ & $3(100.0)$ \\
ESBL . pneumoniae & $2(8.3)$ & $0(0)$ \\
Reassessment $\left(\mathrm{n}_{\text {mother }}=148, \mathrm{n}_{\text {infant }}=151\right)$ & $26(17.6)$ & $24(15.9)$ \\
ESBL E. coli & $26(100.0)$ & $17(70.8)$ \\
ESBL K. pneumoniae & $0(0)$ & $4(16.7)$ \\
CRE K. pneumoniae & $0(0)$ & $4(16.7)$ \\
Overall ( $\left.\mathrm{n}_{\text {mother }}=347, \mathrm{n}_{\text {infant }}=350\right)$ & $50(14.4)$ & $27(7.7)$ \\
ESBL E. coli & $48(96.0)$ & $20(71.4)$ \\
ESBL . p. pneumoniae & $2(4.0)$ & $4(14.8)$ \\
CRE K. pneumoniae & $0(0)$ & $4(14.8)$ \\
\hline
\end{tabular}

Values are reported as frequency (proportion) of mothers or infants who were colonized by at least one isolate from a given category

E. coli: Escherichia coli; K. pneumoniae: Klebsiella pneumoniae; ESBL: extended spectrum $\beta$-lactamase; CRE: carbapenem resistant Enterobacteriaceae detected at reassessment (Fig. 1, Table 2). Antibiotic susceptibility testing results of MDRE isolates are documented in Additional File 1: Table S3. At enrollment and reassessment, respectively, $2 / 3$ (66.7\%) and 9/24 (37.5\%) of MDRE-colonized infants also had MDREcolonized mothers. Of the MDRE-colonized women at enrollment, 22/24 (91.7\%) were colonized with ESBLproducing E. coli and 2/24 (8.3\%) were colonized with ESBL-producing Klebsiella pneumoniae. Of the MDREcolonized women at reassessment, all $(26,100 \%)$ were colonized with ESBL-producing E. coli. All MDREcolonized infants at enrollment $(3,100 \%)$ had ESBLproducing E. coli. Of the MDRE-colonized infants at reassessment, $17 / 24(70.8 \%)$ had ESBL-producing $E$. coli, 4/24 (16.7\%) had ESBL-producing K. pneumoniae, and 4/24 (16.7\%) had carbapenem-resistant K. pneumoniae. One infant at reassessment had both an ESBLproducing and carbapenem-resistant $K$. pneumoniae isolate. The modified Hodge test was negative in all CRE isolates. 


\section{Risk factors for MDRE colonization}

On unadjusted analysis, there were no significant risk factors associated with mothers' or infants' intestinal colonization with MDRE at enrollment. However, mothers were more likely to have intestinal colonization with MDRE at reassessment if they had more children at home $(p=0.001)$ (Additional File 1: Table S1). Infants were more likely to be intestinally colonized with MDRE at reassessment if they had been delivered by $\mathrm{C}$-section $(p=0.021)$, had low birth weight $(p=0.023)$, or if the mother had intestinal colonization with MDRE at enrollment or reassessment ( $p=0.026$ and $p=0.012$, respectively). Multivariable analysis indicated that mother's colonization at enrollment ( $\mathrm{aOR}=3.62,95 \%$ confidence interval $(\mathrm{CI})=1.04-12.57)$, mother's colonization at reassessment $(\mathrm{aOR}=4.44, \mathrm{CI}=1.38-14.30)$, delivery by $\mathrm{C}$-section $(\mathrm{aOR}=2.91, \mathrm{CI}=1.00-8.47)$, and low birth weight $(\mathrm{aOR}=5.39, \mathrm{CI}=1.43-20.27)$ were positively associated with the infant's intestinal colonization (Table 3). Complications during pregnancy or delivery, admission to the NICU, antibiotic use, source of

Table 3 Multivariable analysis of risk factors associated with intestinal colonization with multidrug-resistant Enterobacteriaceae (MDRE) was conducted for infants at reassessment

\begin{tabular}{ll}
\hline Risk factor & Adjusted odds ratio (95\% Cl) \\
\hline Mother colonized at enrollment & $3.62(1.04-12.57)$ \\
Mother colonized at reassessment & $4.44(1.38-14.30)$ \\
Infant colonized at enrollment & $14.2(0.82-245.22)$ \\
C-section delivery & $2.91(1.00-8.47)$ \\
Low birth weight & $5.39(1.43-20.27)$
\end{tabular}

nutrition, and water and animal exposures at home were not significantly associated with MDRE colonization.

\section{Molecular analysis}

Given funding restrictions, only $E$. coli isolates were selected for whole-genome sequencing. Of the $E$. coli isolates from mothers and infants, $47 / 54$ (87.0\%) from mothers and 20/22 (90.9\%) from infants were selected for analysis; the remainder of $E$. coli isolates (4 in mothers and 2 in infants) were omitted due to poor DNA quality or were duplicates ( 3 in mothers). Analysis using the NCBI AMRfinder and Resfinder databases resulted in the identification of 61 genes that conferred resistance to antibiotics, as detailed in Additional File 1: Table S4. The five most common antibiotic resistance-encoding genes found in both mothers and infants were blaEC, blaCTX-M-15, gyrA-S83L, $m p h(\mathrm{~A})$, and blaTEM-1. The median number of resistance genes reported for an isolate was 11 (IQR 7-16) and all isolates sequenced for mothers and infants had at least one ESBL-producing gene. Additionally, in the 47 isolates from mothers, genes encoding resistance to other classes of antibiotics were common: 38 (80.9\%) to quinolones, $33(70.2 \%)$ to fosfomycins, 28 (59.6\%) to aminoglycosides or trimethoprim, 27 (57.5\%) to sulfonamides, and 25 (53.2\%) to macrolides (Table 4). Of note, one of the mother's isolates carried the mcr1.1 gene that encodes resistance to colistin, which was further confirmed by phenotypic screening. Of the 20 isolates from infants, $16(80.0 \%)$ had genes encoding resistance to fosfomycin or trimethoprim and $15(75.0 \%)$ had genes encoding resistance to quinolones or aminoglycosides, and $14(70.0 \%)$ had genes encoding resistance to macrolides.

Table 4 Summary of antimicrobial resistance present in E. coli isolates found in mothers and infants

\begin{tabular}{|c|c|c|c|c|c|c|}
\hline & \multicolumn{3}{|c|}{ Isolates from infants } & \multicolumn{3}{|c|}{ Isolates from mothers } \\
\hline & Overall $n=20$ & Admissions $n=2$ & Reassessment $n=18$ & Overall $n=47$ & Admissions $n=21$ & Reassessment $\mathrm{n}=26$ \\
\hline Aminoglycosides & $15(75.0)$ & $1(50.0)$ & $14(83.3)$ & $28(59.6)$ & $10(47.6)$ & $18(69.2)$ \\
\hline Antiseptics & $10(50.0)$ & $1(50.0)$ & $9(50.0)$ & $20(42.6)$ & $10(47.6)$ & $10(38.5)$ \\
\hline$\beta$-lactams & $20(100.0)$ & $2(100.0)$ & $18(100.0)$ & $47(100.0)$ & $21(100.0)$ & $26(100.0)$ \\
\hline Chloramphenicol & $4(20.0)$ & $1(50.0)$ & $3(16.8)$ & $14(29.8)$ & $6(28.6)$ & $8(30.8)$ \\
\hline Colistin & $0(0)$ & $0(0)$ & $0(0)$ & $1(2.1)$ & $0(0)$ & $1(3.9)$ \\
\hline Fosfomycin & $16(80.0)$ & $2(100.0)$ & $14(77.8)$ & $33(70.2)$ & $13(61.9)$ & $20(76.9)$ \\
\hline Macrolides & $14(70.0)$ & $1(50.0)$ & $13(72.2)$ & $25(53.2)$ & $11(52.4)$ & $14(53.9)$ \\
\hline Quinolones & $15(75.0)$ & $2(100.0)$ & $13(72.2)$ & $38(80.9)$ & $18(85.7)$ & $20(76.9)$ \\
\hline Sulfonamides & $11(55.0)$ & $1(50.0)$ & $10(55.6)$ & $27(57.5)$ & $12(57.1)$ & $15(57.7)$ \\
\hline Tetracyclines & $11(55.0)$ & $1(50.0)$ & $10(55.6)$ & $19(40.4)$ & $9(42.9)$ & $10(38.5)$ \\
\hline Trimethoprim & $16(80.0)$ & $1(50.0)$ & 15 (83.3) & 28 (59.6) & $13(61.9)$ & $15(57.7)$ \\
\hline
\end{tabular}

Values reported as frequency (proportion) of $E$. coli isolates detected carrying the relevant resistance genes. Note that more than one isolate was cultured from some participants and that four isolates from mothers and two isolates from infants were omitted due to poor DNA quality. See Additional File 1: Table S4 for specific genes 


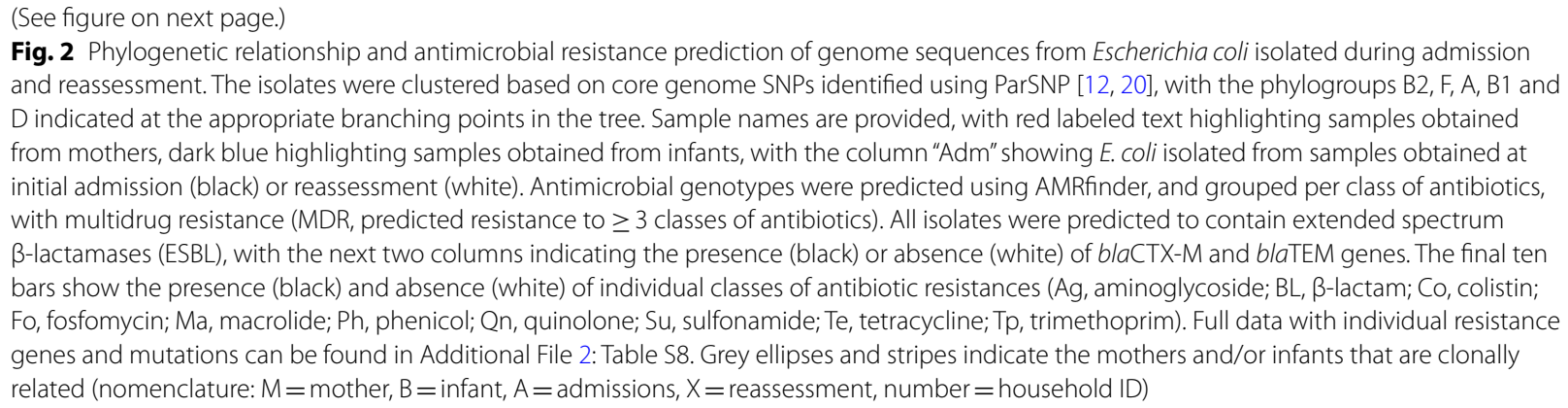

The sequenced isolates fell into five phylogroups (A, B1, B2, D, and F), (Fig. 2, Additional File 1: Tables S5S7). Phylogroup B2 was the most common overall (34\% and $50 \%$ of sequenced isolates from mothers and infants, respectively), followed by phylogroups $\mathrm{F}, \mathrm{D}, \mathrm{A}$, and $\mathrm{B} 1$. Multilocus sequence typing (MLST) revealed that isolates from phylogroup B2 were mostly ST131 (56.3\% and $80.0 \%$ of phylogroup B2 found in mothers and infants, respectively), phylogroup D were mostly ST38 ( $56.3 \%$ and $50.0 \%$ of phylogroup D in mothers and infants, respectively), and phylogroup F were mostly ST648 (50.0\% and $50.0 \%$ of phylogroup $\mathrm{F}$ in mothers and infants, respectively). Of the 20 sequenced isolates from infants, 6 (30\%) were clustered on the same branch as the mother's isolates, 10 (50.0\%) isolates were from infants whose mothers were not detected to be colonized with multidrug-resistant E. coli, and 4 (20.0\%) were clustered on branches different from the mother's isolate. The motherinfant pairs with isolates clustered on the same branch were distributed evenly across all phylogroups (except for phylogroup B1, which had none), suggesting there was no single strain that was more likely to be transmitted from mother to infant. Of the infants colonized at enrollment, $2 / 3(66.7 \%)$ had a similar isolate detected at reassessment, suggesting that early colonization may not be transient. Infants colonized at reassessment (whose mothers were not colonized) were distributed across the phylogroups and had varied resistance profiles, further suggesting that there was not a common source for or strain of MDREs.

\section{Discussion}

Understanding how and when intestinal colonization occurs is important for designing control strategies that reduce MDRE colonization prevalence. Of interest is the Middle East and South Asia, where MDRE colonization prevalence is higher than in other parts of the world but not widely studied. We present the first report on prevalence of MDRE colonization in Sri Lanka, where a high (12-17.6\%) proportion of women were colonized with MDRE. While only $1.5 \%$ of infants were colonized with MDRE at birth, this number increased to $15.9 \%$ by 6 weeks of age. Our study is hypothesis generating, and suggests that postnatal MDRE colonization via contact transmission in the community, including from mother's kissing, skin-to-skin contact, or breastmilk, may be more common than intranatal colonization for infants.

The prevalence of intestinal MDRE colonization was high in women in this study, relative to Western settings (12-17.6\% vs $1-6 \%$, respectively) [22-24]. The reason for the higher prevalence in our study is likely multi-factorial, but different practices in antibiotic use and access may be important. One study showed that the majority $(80 \%)$ of outpatients with likely viral respiratory illnesses in Sri Lanka were prescribed antibiotics [25]. Similarly, a survey of pharmacy students in Sri Lanka reported that $76 \%$ of them had used antibiotics in the last year [26]. This overuse of antibiotics is driven by multiple factors, including physicians' perception that patients desire antibiotics, clinicians' inability to discern viral from bacterial infections based on symptoms, and patients' ability to obtain over-the-counter antibiotics [26, 27]. Our intestinal colonization prevalence in women is similar to figures reported previously from some studies in low- and middle-income countries (India 15\% ESBL-E colonization in 2007-2009, Tanzania 15\% ESBL-E colonization in 2013, and Madagascar 18.5\% ESBL-E colonization in 2014) [3, $6,9,28]$.

The observation of colonization of infants within 6 weeks after birth in this study corroborates limited data from other studies in areas of high MDRE colonization prevalence [10, 29]. A study set in India showed that infant colonization with ESBL-E increased from 14.3\% after birth to $41.5 \%$ in 60 days [10]. A study set in Spain reported that $19.6 \%$ of infants with ESBL-E-colonized mothers were colonized with ESBL-E at birth and 22.2\% were colonized after 3 months [29]. The latter number decreased to $11.1 \%$ after 1 year. Overall, these prevalence rates are high and reflective of the level of endemicity in a given community. Relative to these studies, we reported a lower initial colonization prevalence in infants, possibly 


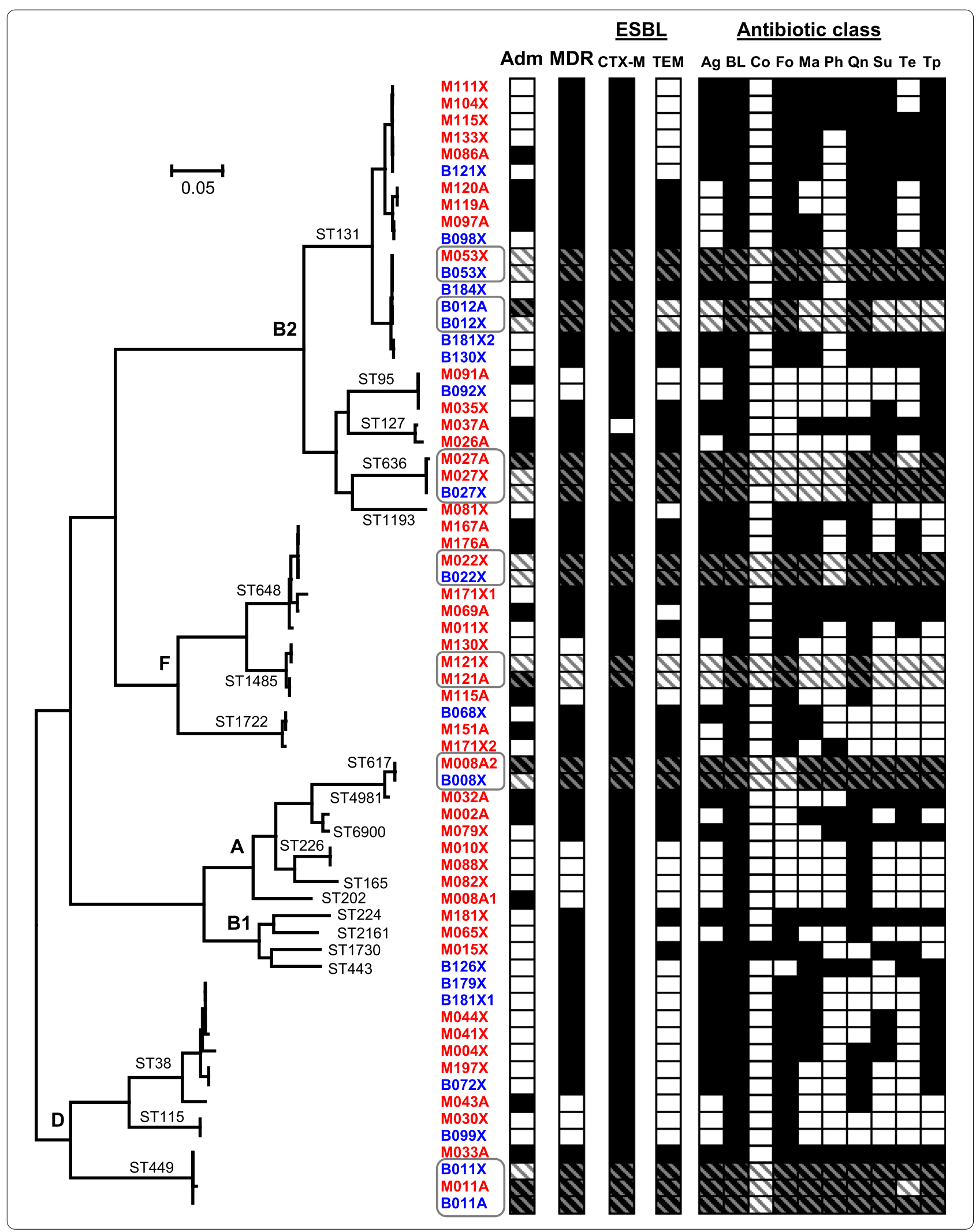


reflective of a lower colonization prevalence in mothers; however, we saw a similar rapid colonization in the weeks following birth.

Colonization of mothers as a significant factor in infant colonization corroborates data from other studies [5, 29]. Infants delivered vaginally are exposed to their mothers' intestinal microbiota, including MDRE that may be present [30]. Infants delivered by $\mathrm{C}$-section had a higher risk of being colonized with MDRE at reassessment. Since women who delivered by $\mathrm{C}$-section received a prophylactic antibiotic, the risk could be due to the selective pressure of the antibiotic and/or the exposure to a different microbiome. As infants delivered by $\mathrm{C}$-section are colonized by different bacteria than those delivered vaginally, it could be easier for MDRE to become established in their microbiome [31, 32]. Additionally, studies have shown that intestinal bacteria can be present in breastmilk, posing another way by which an infant may be exposed to a mother's microbiome [32, 33].

Genotyping revealed that a quarter of colonized infants were colonized with a strain found in the same cluster as their mother's isolate and more than half of colonized infants at reassessment did not have colonized mothers. This finding supports data from other studies that found little-to-no similarity in ESBL isolates between mothers and their infants $[9,29]$. All of the sequenced MDRE isolates carried CTX-M genes and about one-third harbored OXA genes, conferring resistance to a wide range of $\beta$-lactam antibiotics. This finding is consistent with observations in other South and Southeast Asian countries that have reported a high prevalence of CTX-M and OXA enzymes [34, 35]. Interestingly, we did not detect OXA-23, reportedly the most prevalent oxacillinase today. In addition to $\beta$-lactam resistance, genotyping and phenotypic screening detected isolates with resistance to a wide variety of other antibiotics. A large proportion of isolates were resistant to quinolones, supporting previous findings that ESBL production is often associated with quinolone resistance [36]. Isolates were also detected with resistance to colistin and fosfomycin, which are considered antibiotics of last resort in most settings [37]. To our knowledge, this is the first time colistin and fosfomycin resistance have been reported in Sri Lanka, and this finding is further evidence of the worrisome rise in antibiotic resistance.

Molecular typing of E. coli isolates revealed that phylogroup B2 was the most common among our isolates, with ST131 being the predominant sequence type within this group. Our findings are consistent with data from around the world which suggest that phylogroup B2 and ST131 are prevalent among ESBL-producing E. coli globally [38]. E. coli ST131 in particular has spread throughout the world, commonly carrying the ESBL-encoding gene
CTX-M-15 and encoding multidrug resistance [39]. In our study, all of our ST131 isolates carried this antibiotic resistance gene.

Some limitations in our study must be noted. Single rectal swabs may have limited the ability to detect MDRE isolates and may have led to low estimates of relatedness between individuals [40]. Consequently, the ability to accurately characterize transmission routes and common sources of MDRE (either hospital or community) was limited. Variations in single swab detection sensitivity could explain the discrepancy observed with some mothers being colonized only at enrollment or reassessment (not both) and could lead to an underestimation of MDRE intestinal colonization. Repeated swabs, using an enriched broth detection method, or additional time points would increase the chances of capturing a MDRE isolate and shed light on the degree to which MDRE colonization is transient [41]. Another source of MDRE prevalence underestimation may have been that some isolates initially grew on the MacConkey and cefotaxime selective media but could not be re-cultured. This loss of isolates could be due to decreased viability of organisms following a freeze-thaw cycle. However, a sensitivity analysis assessing for risk factors associated with MDRE colonization showed no changes from the primary analysis when these lost isolates were considered as MDRE. Ultimately, the loss of isolates during the storage process or during the freeze-thaw cycle would only have underestimated the number of women and infants reported to be colonized with MDRE. We were only able to perform reassessments on $75 \%$ of subjects, and we did not follow patients beyond 6 weeks after enrollment. While the modified Hodge test that we performed was recommended by the CLSI for phenotypic confirmation of carbapenemase production, other tests, such as the Carba NP test or modified Carbapenemase Inactivation Method (mCIM), have improved specificity and sensitivity and could have provided a more sensitive method for detecting carbapenemases. Finally, the study's limited funding precluded whole genome sequencing of the K. pneumoniae isolates; thus, the antibiotic resistance genes, phylogroups, and relatedness between mother and infant of these MDRE remain to be characterized. In addition, while our study has highlighted a high prevalence of MDRE intestinal colonization, the association of MDRE colonization with subsequent infection needs to be further characterized.

\section{Conclusion}

In conclusion, MDRE intestinal colonization was established within 6 weeks of birth among infants in Sri Lanka. Our results suggest that infants were likely colonized during the first few weeks after birth, possibly due to 
colonization from an intranatal or nosocomial exposure, contact transmission in the community, or breastmilk. Identifying how and when MDRE colonization occurs will be critical for preventing such colonization, which may be a precursor to MDRE infections that are challenging to treat.

\section{Abbreviations}

MDRE: Multidrug-resistant Enterobacteriaceae; ESBL-E: Extended-Spectrum $\beta$-lactamase-producing Enterobacteriaceae; CRE: Carbapenem-Resistant Enterobacteriaceae.

\section{Supplementary Information}

The online version contains supplementary material available at https://doi. org/10.1186/s13756-021-00938-3.

Additional file 1: Table S1. Description of mothers at enrollment and reassessment in addition to bivariable analysis of risk factors associated with intestinal colonization with multidrug resistant Enterobacteriaceae (MDRE). Table S2. Sensitivity analysis for definition of isolates that were not re-culturable. Table S3. Summary of antibiotic resistance by phenotypic susceptibility testing of isolates found in mothers and infants. Table S4. Summary of antibiotic resistance-encoding genes present in isolates found in mothers and infants. Table S5. Information on E. coli isolates characterized using whole genome sequencing. Table $\mathbf{S 6}$ Mothers' isolates categorized by phylogroup and Multilocus Sequence Typing (MLST). Table S7. Infants' isolates categorized by phylogroup and Multilocus Sequence Typing (MLST).

Additional file 2: Table S8. Antimicrobial resistance prediction of E. coli genomes included in this study, as determined by AMRfinder.

\section{Acknowledgements}

We would like to thank the patients and research staff who were involved in this study.

\section{Authors' contributions \\ HRM, SK, AN, CB, RK, BP, BW, BPN, CWW, and LGT contributed to study design SK, RK, NW, BP, and LGT collected the data. KN, LH, AH, and MJ conducted laboratory testing. ST and LH conducted whole genome sequencing and data analysis. HRM, BW, and BPN contributed to statistical analysis. HRM, AHMV, and LGT analyzed and interpreted the results. CWW provided oversight of testing and analysis and LGT conceived the study. HRM and LGT wrote the paper. All authors read and approved the final manuscript.}

\section{Funding}

This study was supported by funds from the Triangle Center for Evolutionary Medicine (TriCEM). HRM received a travel grant from the Duke Global Health Institute. LGT is supported by the National Institute of Allergy and Infectious Diseases (K23Al125677). Site support is provided by the Duke Global Health Institute and the Duke Hubert-Yeargan Center for Global Health. The whole genome sequencing was completed by the FDA GenomeTrakr program funded grant 1U18FD00678801.

\section{Availability of data and materials}

Data from the surveys and microbiological testing are available upon request. The final genomic sequences were uploaded to the designated NCBI Bioproject (PRJNA293225).

\section{Declarations}

\section{Ethics approval and consent to participate}

Ethical approval for this study was obtained from the Faculty of Medicine, University of Ruhuna, Ethical Review Committee (Sri Lanka) and the Duke University Institutional Review Board (USA).
Consent for publication

Not applicable.

\section{Competing interests}

The authors declare that they have no competing interests.

\section{Author details}

${ }^{1}$ Department of Biomedical Engineering, Duke University, Durham, NC, USA. ${ }^{2}$ Department of Epidemiology, Johns Hopkins Bloomberg School of Public Health, Baltimore, MD, USA. ${ }^{3}$ Department of Obstetrics and Gynecology, Faculty of Medicine, University of Ruhuna, Galle, Sri Lanka. ${ }^{4}$ Division of Infectious Diseases, Department of Medicine, Duke University School of Medicine, Durham, NC, USA. ${ }^{5}$ Department of Microbiology, Faculty of Medicine, University of Ruhuna, Galle, Sri Lanka. ${ }^{6}$ Duke Global Health Institute, Duke University, Durham, NC, USA. ${ }^{7}$ Department of Medicine, Faculty of Medicine, University of Ruhuna, Galle, Sri Lanka. ${ }^{8}$ Teaching Hospital Karapitiya, Galle, Sri Lanka. ${ }^{9}$ Department of Entomology and Plant Pathology, NC State University, Raleigh, NC, USA. ${ }^{10}$ Institute of Medical Research, Durham, NC, USA. ${ }^{11}$ Durham Veterans Affairs Health System, Durham, NC, USA. ${ }^{12}$ Department of Pathology and Infectious Diseases, School of Veterinary Medicine, University of Surrey, Guildford, UK. ${ }^{13}$ Department of Population Health and Pathobiology, NC State University, Raleigh, NC, USA. ${ }^{14}$ Comparative Medicine Institute, NC State University, Raleigh, NC, USA.

Received: 13 December 2020 Accepted: 21 April 2021

Published online: 30 April 2021

\section{References}

1. Laxminarayan R, Duse A, Wattal C, Zaidi AKM, Wertheim HFL, Sumpradit N, Vlieghe E, Hara GL, Gould IM, Goossens H, Greko C, So AD, Bigdeli M, Tomson G, Woodhouse W, Ombaka E, Peralta AQ, Qamar FN, Mir F, Kariuki S, Bhutta ZA, Coates A, Bergstrom R, Wright GD, Brown ED, Cars O. Antibiotic resistance-the need for global solutions. Lancet Infect Dis. 2013;13:1057-98

2. Centers for Disease Control and Prevention. 2013. Antibiotic Resistance Threats in the United States.

3. Woerther P-L, Burdet C, Chachaty E, Andremont A. Trends in human fecal carriage of extended-spectrum-lactamases in the community: toward the globalization of CTX-M. Clin Microbiol Rev. 2013;26:744-58.

4. McConville TH, Sullivan SB, Gomez-Simmonds A, Whittier S, Uhlemann AC. Carbapenem-resistant Enterobacteriaceae colonization (CRE) and subsequent risk of infection and 90-day mortality in critically ill patients, an observational study. PLoS ONE. 2017;12(10):e0186195.

5. Denkel LA, Schwab F, Kola A, Leistner R, Garten L, von Weizsäcker K, Geffers C, Gastmeier P, Piening B. The mother as most important risk factor for colonization of very low birth weight (VLBW) infants with extended-spectrum b-lactamase-producing Enterobacteriaceae (ESBL-E). J Antimicrob Chemother. 2014;69:2230-7.

6. Chereau F, Herindrainy P, Garin B, Huynh B-TT, Randrianirina F, Padget M, Piola P, Guillemot D, Delarocque-Astagneau E. Colonization of extendedspectrum- $\beta$-lactamase- and NDM-1-producing Enterobacteriaceae among pregnant women in the community in a low-income country: a potential reservoir for transmission of multiresistant Enterobacteriaceae to neonates. Antimicrob Agents Chemother. 2015;59:3652-5.

7. Meyer E, Gastmeier P, Kola A, Schwab F. Pet animals and foreign travel are risk factors for colonisation with extended-spectrum $\beta$-lactamaseproducing Escherichia coli. Infection. 2012;40:685-7.

8. Babu R, Kumar A, Karim S, Warrier S, Nair SG, Singh SK, Biswas R. Faecal carriage rate of extended-spectrum $\beta$-lactamase-producing Enterobacteriaceae in hospitalised patients and healthy asymptomatic individuals coming for health check-up. J Glob Antimicrob Resist. 2016;6:150-3.

9. Nelson E, Kayega J, Seni J, Mushi MF, Kidenya BR, Hokororo A, Zuechner A, Kihunrwa A, Mshana SE. Evaluation of existence and transmission of extended spectrum beta lactamase producing bacteria from post-delivery women to neonates at Bugando Medical Center, Mwanza-Tanzania. BMC Res Notes. 2014;7:1-6.

10. Kothari C, Gaind R, Singh LC, Sinha A, Kumari V, Arya S, Chellani H, Saxena S, Deb M. Community acquisition of $\beta$-lactamase producing Enterobacteriaceae in neonatal gut. BMC Microbiol. 2013;13:1-6. 
11. CLSI. Performance Standards for Antimicrobial Susceptibility Testing. 27th ed. Wayne: Clinical and Laboratory Standards Institute; 2017.

12. Pornsukarom S, Van Vliet AHM, Thakur S. Whole genome sequencing analysis of multiple Salmonella serovars provides insights into phylogenetic relatedness, antimicrobial resistance, and virulence markers across humans, food animals and agriculture environmental sources. BMC Genomics. 2018;19:1-14.

13. Bankevich A, Nurk S, Antipov D, Gurevich AA, Dvorkin M, Kulikov AS, Lesin VM, Nikolenko SI, Pham S, Prjibelski AD, Pyshkin AV, Sirotkin AV, Vyahhi N, Tesler G, Alekseyev MA, Pevzner PA. SPAdes: a new genome assembly algorithm and its applications to single-cell sequencing. J Comput Biol. 2012;19:455-77.

14. Gurevich A, Saveliev V, Vyahhi N, Tesler G. Genome analysis QUAST: quality assessment tool for genome assemblies. Bioinformatics. 2013;29:1072-5.

15. Wirth T, Falush D, Lan R, Colles F, Mensa P, Wieler LH, Karch H, Reeves PR, Maiden MCJ, Ochman H, Achtman M. Sex and virulence in Escherichia coli: an evolutionary perspective. Mol Microbiol. 2006;60:1136-51.

16. Joensen KG, Tetzschner AMM, Iguchi A, Aarestrup FM, Scheutz F. Rapid and easy in silico serotyping of Escherichia coli isolates by use of wholegenome sequencing data. J Clin Microbiol. 2015;53:2410-26.

17. Beghain J, Bridier-Nahmias A, Nagard H Le, Denamur E, Clermont O. ClermonTyping: an easy-to-use and accurate in silico method for Escherichia genus strain phylotyping. Microb Genom. 2018;4(7):e000192.

18. Feldgarden M, Brover V, Haft DH, Prasad AB, Slotta DJ, Tolstoy I, Tyson GH, Zhao S, Hsu CH, McDermott PF, Tadesse DA, Morales C, Simmons M, Tillman G, Wasilenko J, Folster JP, Klimke W. Validating the AMRFINder tool and resistance gene database by using antimicrobial resistance genotype-phenotype correlations in a collection of isolates. Antimicrob Agents Chemother. 2019;63:e00483-19.

19. Zankari E, Hasman H, Cosentino S, Vestergaard M, Rasmussen S, Lund O, Aarestrup FM, Larsen MV. Identification of acquired antimicrobial resistance genes. J Antimicrob Chemother. 2012;67:2640-4.

20. Treangen TJ, Ondov BD, Koren S, Phillippy AM. The harvest suite for rapid core-genome alignment and visualization of thousands of intraspecific microbial genomes. Genome Biol. 2014;15:1-15.

21. Indicative US Dollar SPOT Exchange Rate (LKR per 1 USD) | Central Bank of Sri Lanka.

22. Valenza G, Nickel S, Pfeifer Y, Eller C, Krupa E, Lehner-Reindl V, Höller C. Extended-spectrum- $\beta$-lactamase-producing Escherichia coli as intestinal colonizers in the German community. Antimicrob Agents Chemother. 2014;58:1228-30.

23. Harris AD, Nemoy L, Johnson JA, Martin-Carnahan A, Smith DL, Standiford $\mathrm{H}$, Perencevich EN. Co-carriage rates of vancomycin-resistant enterococcus and extended-spectrum beta-lactamase-producing bacteria among a cohort of intensive care unit patients: implications for an active surveillance program. Infect Control Hosp Epidemiol. 2004;25:105-8.

24. Valverde A, Coque TM, Sánchez-Moreno MP, Rollán A, Baquero F, Cantón R. Dramatic increase in prevalence of fecal carriage of extended-spectrum $\beta$-lactamase-producing Enterobacteriaceae during nonoutbreak situations in Spain. J Clin Microbiol. 2004;42:4769-75.

25. Tillekeratne LG, Bodinayake CK, Nagahawatte A, Vidanagama D, Devasiri V, Arachchi WK, Kurukulasooriya R, De Silva AD, Østbye T, Reller ME, Woods CW. Use of rapid influenza testing to reduce antibiotic prescriptions among outpatients with influenza-like illness in southern Sri Lanka. Am J Trop Med Hyg. 2015;93:1031-7.

26. Sakeena MHF, Bennett AA, Jamshed S, Mohamed F, Herath DR, Gawarammana I, McLachlan AJ. Investigating knowledge regarding antibiotics and antimicrobial resistance among pharmacy students in Sri Lankan universities. BMC Infect Dis. 2018;18:1-11.
27. Tillekeratne LG, Bodinayake CK, Dabrera T, Nagahawatte A, Arachchi WK, Sooriyaarachchi A, Stewart K, Watt M, Østbye T, Woods CW. Antibiotic overuse for acute respiratory tract infections in Sri Lanka: a qualitative study of outpatients and their physicians. BMC Fam Pract. 2017;18:1-10.

28. Pathak A, Chandran SP, Mahadik K, Macaden R, Lundborg CS. Frequency and factors associated with carriage of multi-drug resistant commensal Escherichia coli among women attending antenatal clinics in Central India. BMC Infect Dis. 2013;13:1-9.

29. Rodríguez-Revuelta MJ, López-Cerero L, Serrano L, Luna-Lagares S, Pascual A, Rodríguez-Baño J. Incidence and risk factors for acquisition of extended-spectrum $\beta$-lactamase-producing enterobacteriaceae in newborns in Seville, Spain: a prospective cohort study. Int J Antimicrob Agents. 2018;52:835-41.

30. Houghteling PD, Walker WA. Why is initial bacterial colonization of the intestine important to infants' and children's health? J Pediatr Gastroenterol Nutr. 2015;60:294-307.

31. Dominguez-Bello MG, Costello EK, Contreras M, Magris M, Hidalgo G, Fierer N, Knight R. Delivery mode shapes the acquisition and structure of the initial microbiota across multiple body habitats in newborns. Proc Natl Acad Sci U S A. 2010;107:11971-5.

32. Wang S, Ryan CA, Boyaval P, Dempsey EM, Ross RP, Stanton C. Maternal vertical transmission affecting early-life microbiota development. Trends Microbiol. 2020;28:28-45.

33. Perez PF, Leclerc M, Levenez F, Benyacoub J, Serrant P, SeguraRoggero I, Schiffrin EJ, Donnet-Hughes A. Bacterial imprinting of the neonatal immune system: lessons from maternal cells? Pediatrics. 2007:119:e724-32.

34. Hawkey PM. Prevalence and clonality of extended-spectrum beta-lactamases in Asia. Clin Microbiol Infect. 2008;14:159-65.

35. Hsu LY, Apisarnthanarak A, Khan E, Suwantarat N, Ghafur A, Tambyah P. Carbapenem-resistant acinetobacter baumannii and enterobacteriaceae in South and Southeast Asia. Clin Microbiol Rev. 2017;30:1-22.

36. Lautenbach E, Strom BL, Bilker WB, Patel JB, Edelstein PH, Fishman NO. Epidemiological investigation of fluoroquinolone resistance in infections due to extended-spectrum²-lactamase-producing Escherichia coli and Klebsiella pneumoniae. Clin Infect Dis. 2001;33:1288-94.

37. Falagas ME, Karageorgopoulos DE, Nordmann P. Therapeutic options for infections with Enterobacteriaceae producing carbapenem-hydrolyzing enzymes. Future Microbiol. 2011;6:659-66.

38. Nicolas-Chanoine MH, Blanco J, Leflon-Guibout V, Demarty R, Alonso MP, Caniça MM, Park YJ, Lavigne JP, Pitout J, Johnson JR. Intercontinental emergence of Escherichia coli clone O25:H4-ST131 producing CTX-M-15. J Antimicrob Chemother. 2008;61:273-81.

39. Nicolas-Chanoine MH, Bertrand X, Madec JY. Escherichia coli st131, an intriguing clonal group. Clin Microbiol Rev. 2014;27:543-74.

40. Worby CJ, Lipsitch M, Hanage WP. Within-host bacterial diversity hinders accurate reconstruction of transmission networks from genomic distance data. PLoS Comput Biol. 2014;10:1003549.

41. Murk JLAN, Heddema ER, Hess DLJ, Bogaards JA, Vandenbroucke-Grauls CMJE, Debets-Ossenkopp YJ. Enrichment broth improved detection of extended-spectrum-beta-lactamase- producing bacteria in throat and rectal surveillance cultures of samples from patients in intensive care units. J Clin Microbiol. 2009:47:1885-7.

\section{Publisher's Note}

Springer Nature remains neutral with regard to jurisdictional claims in published maps and institutional affiliations. 